\title{
Virtudes y fortalezas de carácter en personas recluidas en un sistema penitenciario
}

\section{Virtues and Character Strengths in persons detained in a penitentiary system}

Anais Ortiz Valdez

Víctor Corral Verdugo

Universidad de Sonora

\section{Resumen}

Dentro del campo de estudio de la psicología de la criminalidad, con cierta regularidad se caracteriza a las personas que han cometido algún delito como individuos con rasgos de personalidad negativos y carentes de fortalezas de carácter. Sin embargo, la Psicología Positiva (PP) plantea la noción de que todos los seres humanos poseen, en grados variables, virtudes y fortalezas y que éstas se manifiestan universalmente en la mayoría de las culturas. El objetivo del presente estudio fue determinar y analizar si las personas que han cometido algún delito presentan, por lo tanto, rasgos virtuosos y fortalezas de carácter. El estudio se llevó a cabo en una institución penitenciaria de la ciudad de Hermosillo, Sonora, México. Se aplicó el inventario de virtudes y fortalezas (VIA-S), en su versión del idioma español y reducido a una muestra de 104 internos: $89.4 \%$ hombres y 10.6\% mujeres. A partir de los hallazgos se concluyó que, así como el resto de las personas, los individuos que han cometido algún acto delictivo poseen rasgos virtuosos y gozan de fortalezas de carácter.

Palabras clave: virtudes, fortalezas, delincuentes.

\begin{abstract}
Within the field of criminal psychology, people who have committed crimes often are characterized as having negative personality traits and lacking character strengths. Yet, Positive Psychology states that all human beings possess, to certain extent, virtues and strengths and these are universally manifested in most cultures. This paper is aimed at determining and analyzing whether individuals who have committed a crime exhibit virtues and character strengths. The study was conducted in a penitentiary

Nota del autor

Anais Ortiz Valdez, Doctorado en Ciencias Sociales, Universidad de Sonora (UNISON); Victor Corral Verdugo, Posgrado Integral en Ciencias Sociales, UNISON.

La correspondencia en relación con este artículo debe dirigirse a Anais Ortiz Valdez, Doctorado en Ciencias Sociales, UNISON, bulevar Luis Encinas y Rosales s/n, colonia Centro, C.P. 83000, Hermosillo Sonora, México.

Dirección electrónica: anais.ortizv@gmail.com
\end{abstract}


institution at Hermosillo, Mexico. The Virtues and Strengths Inventory (VIA-S), Spanish reduced version, was administered to a sample of 104 inmates: $89.4 \%$ males and $10.6 \%$ females. The findings revealed that, as any other people, individuals who have committed any offense exhibit virtuous traits and character strengths.

Keywords: virtues, strengths, inmates.

Hasta hace algunos años el estudio de las ciencias sociales, como el de la psicología, se enfocaba en la búsqueda de explicaciones y soluciones a los grandes males que aquejan a la sociedad y descuidaba, sustancialmente, la investigación del potencial humano para lograr el bienestar, la felicidad y otros estados psicológicos positivos. En otras palabras, se establecían las causas de la negatividad y se dejaban de lado los determinantes de la positividad. La aproximación negativa en psicología focaliza el estudio y la investigación, casi de manera total, en comportamientos anormales o desajustados. Como respuesta a esa aproximación al estudio del comportamiento y los fenómenos sociales, justamente al final del siglo XX nació una nueva corriente: la Psicología Positiva (PP), que investiga aspectos de la ciencia del comportamiento, en sí, dejados de lado, o bien, los factores benéficos para el ser humano que estimulan su desarrollo; lo que hace que la vida sea lo más satisfactoria posible (Seligman, \& Csikszentmihalyi, 2000).

A pesar del significativo beneficio que representaba la aproximación psicológica positiva, ésta parecía presentar limitaciones, al igual que lo hace el enfoque negativo al querer complementar y corregir el desequilibrio de la psicología tradicional en su sesgo hacia posturas de investigación de las patologías, la PP se decantaba por la positividad y esto dejaba por fuera a grupos de individuos que, hasta hace poco, eran catalogados como ajenos a la "normalidad". En consecuencia, poco se ha estudiado la positividad que puede caracterizar a quienes se involucran en conductas antisociales, $\mathrm{y}$, por ende, se llega a aceptar conclusiones no del todo certeras al caracterizar la conducta humana (Linley, 2009).

Cabe señalar que algunos psicólogos positivos han hecho hincapié no sólo en los rasgos y las experiencias positivas, sino además en las formas en que éstos pueden ser utilizados para abordar problemas. En el caso de la conducta antisocial, se podría estudiar lo positivo de las personas recluidas en instituciones penitenciarias, proporcionar así una alternativa al modelo de negatividad del funcionamiento humano que ha dominado el campo más amplio de la psicología (Seligman, 2011; Seligman, \& Csikszentmihalyi, 2000). Los sesgos que han predominado en la psicología en general pueden conducir a visiones dicotómicas de la realidad ("bueno-malo", "positivo-negativo"), o a conclusiones erróneas respecto a las fuentes de la positividad: "lo bueno siempre viene de 
lo bueno", "de lo malo sólo puede generarse daño" (Corral et al., 2014). Uno de los campos que puede ilustrar este posible sesgo es el del estudio de la relación entre fortalezas de carácter y la conducta antisocial, y hacia él orientaremos el estudio y la discusión en el presente artículo.

En el enfoque de la PP se abordan tópicos como las emociones positivas, la felicidad, las capacidades y virtudes humanas, entre otros. Para su estudio se utiliza una gran variedad de métodos y teorías que funciona de forma objetiva y sistemática, optando por aquellos que trabajan a partir de una base científica (Vera, 2006). Dentro de la PP se desarrolla también la investigación de las conductas que llevan a la conservación del andamiaje social y de la integridad física del ambiente, así como las virtudes psicológicas que están constituidas por factores de personalidad, llamados éstos fortalezas de carácter.

Existen seis virtudes que se manifiestan en casi todas las culturas del mundo: el valor, la humanidad, la justicia, la sabiduría, la trascendencia y la moderación (Dahlsgaard, Peterson, \& Seligman, 2005). Peterson y Seligman han propuesto que cada una de estas virtudes se encuentra formada por un cierto número de fortalezas (la tabla 2 representa las seis virtudes y sus correspondientes fortalezas de carácter).

En los últimos años se ha realizado investigación acerca de la conducta prosocial y su relación con las virtudes y fortalezas de carácter (Dahlsgaard et al., 2005). De manera relacionada, dentro de la visión tradicional de los estudios en criminalidad se ha asumido que las personas virtuosas evitan romper las normas sociales e infringir las leyes. Y, por el contrario, cuando se trata de describir a personas antisociales o que han cometido algún(os) delito(s) se asume que éstas carecen de fortalezas de carácter, con base en la idea de la polarización entre lo "bueno" y lo "malo" en general, además de la idea de virtuoso o no virtuoso como manifestación particular de esa dicotomía. Por ejemplo, se identifica en personas que han cometido un delito la ausencia de fortalezas como la inteligencia general (Moffitt, 1997), la inteligencia social y emocional, (Sharma, Prakash, Sengar, Shaudhury, \& Sing, 2015), la templanza (Pizarro, \& Salovey, 2002), la cooperación y otras fortalezas de la humanidad (National Research Council and Institute of Medicine, 2001). En esencia, de acuerdo con esta visión, la persona que ha cometido un delito carece de muchas o todas las fortalezas de carácter, y dicha carencia explica su orientación hacia lo "malo", de acuerdo con lo que las convenciones sociales definen como conducta ajustada.

Sin embargo, dentro de su marco de estudio la PP sugiere que los seres humanos, sin distinguir entre quienes han cometido algún delito $\mathrm{y}$ quienes no lo han hecho, pueden caracterizarse por poseer virtudes y fortalezas. Lo anterior nos lleva a cuestionar: ¿Se encontrarán los delincuentes carentes de virtudes/fortalezas? 
En la lógica social tradicional se plantea que la virtud se contrapone a la ilegalidad, esto es, las personas virtuosas no rompen o infringen las leyes. Las virtudes se componen de fortalezas de carácter y con ello se asume que hablar de individuos antisociales implica suponer que ellos no poseen esas fortalezas. Quizás esto pueda deberse a que a lo largo del estudio de la personalidad del delincuente se le ha caracterizado con rasgos tales como la agresividad, el egocentrismo, la imprevisión e indiferencia afectiva, además de la carencia de un sentimiento de la injusticia padecida, de insensibilidad con respeto a la vida ajena, de un poder incontrolable, un sadismo de dominaciónnarcisista, con padecimientos de psicosis, neurosis, psicopatías, con carácter nervioso, y un nivel inferior en el razonamiento moral, entre otros (Beltri, \& Fuentes, 2008; Landazabal, 2005; Escrivá, García, \& Navarro, 2002; Romero, 2001; Romi, 1995; Pinatel, 1979; Kohlbert, 1969; Hesnard, 1963; Resten, 1963; Hurwttz, 1956; Greeff, 1950).

A pesar de tal caracterización, en la presente investigación planteamos la idea de que algunos delincuentes podrían poseer muchas de las fortalezas de carácter, y que, de hecho, dichas fortalezas podrían serles de utilidad en la comisión de sus actividades antisociales (es decir, de algo bueno para el individuo antisocial — sus fortalezas - podría resultar algo malo para la sociedad: el crimen). Por ejemplo, del valor, la creatividad, la curiosidad, el gusto por aprender, el liderazgo y el trabajo en equipo, pueden generarse conductas criminales efectivas. Es notorio que cada día surgen nuevas y creativas formas de delincuencia (Temperini, 2014). En el estudio de la identidad de las organizaciones criminales y las redes ilegales se ha demostrado que estos grupos se caracterizan por el liderazgo basado en la posición jerárquica o en la posesión de contactos y recursos, respectivamente (Giménez, Ojea, Nieto, Martínez, \& Fernández, 2012). Así también, en un estudio realizado por Shaw (s/f, citado en Marchiori, 2004), los grupos delictivos poseían horarios de trabajo, descanso, diversión, disciplina, castigo y normas especiales de comportamiento, aun cuando éstas fueran opuestas a la vida de la comunidad general (Maqueda, 2010). Todas estas manifestaciones de comportamiento corresponden a fortalezas de carácter $\mathrm{o}$, por lo menos a un carácter de sentido técnico.

Para quienes forman parte de grupos delictivos es de suma importancia el cuidado y la amistosidad hacia otros (especialmente si son miembros de su banda); componente éste de la virtud de la humanidad. También la trascendencia, otra de las virtudes, es una de las características de los que forman parte de estos grupos, ya sea en su "legado" o en la espiritualidad que profesan (Maqueda, 2010). Además, quienes delinquen utilizan el conocimiento, su curiosidad y mente abierta para poder llevar a cabo comportamientos que se encuentran fuera de la norma, como los 
delincuentes informáticos, algunos de los cuales buscan experimentar nuevas tareas o se imponen retos para demostrar su capacidad (Gacharná, 2009).

\section{Virtudes y fortalezas de carácter}

El significado de las virtudes recae en la capacidad o potencia propia del ser humano de expresar una naturaleza moral (expresada de acuerdo al grupo social en extenso o con el grupo delictivo al que se pertenece, en este caso). Lo anterior en tanto que las virtudes son manifestaciones de "excelencia o fortalezas de carácter que hacen posible a los individuos florecer como seres humanos" (Fowers, 2005).

Para su estudio, de las fortalezas se engloban en factores de segundo orden: virtudes, las cuales se presentan como características universales en todas las culturas, incluyendo la mexicana (Dahlsgaard et al., 2005; Corral, Tapia, Ortiz, \& Fraijo, 2013). Estas virtudes son: el valor, la moderación, la trascendencia, la justicia, la humanidad y la sabiduría o conocimiento (Dahlsgaard et al., 2005).

Dentro de las seis virtudes psicológicas identificadas hay fortalezas particulares que se asocian a ellas y se definen como rasgos positivos que se identifican en pensamientos, sentimientos y conductas. Así también las fortalezas pueden medirse como diferencias individuales y se manifiestan en grados variables o distintos.

En la PP, la virtud de la sabiduría es entendida por Peterson y Seligman (2004) como el conjunto de fortalezas cognitivas que permiten adquirir y usar el conocimiento, e incluye la creatividad, curiosidad, mentalidad abierta, amor por aprender y perspectiva como sus fortalezas particulares.

La virtud del valor, también denominada "coraje", comprende un conjunto de fortalezas emocionales que involucra el ejercicio de la voluntad para cumplir objetivos, al encarar la oposición externa o interna (Peterson, \& Seligman, 2004). Dicha virtud está compuesta por cuatro fortalezas particulares: la autenticidad, la valentía, la persistencia y el entusiasmo.

La virtud de la humanidad cobija fortalezas interpersonales que involucran el cuidado y la amistosidad hacia otros (Peterson, \& Seligman, 2004). Las fortalezas particulares de esta virtud son la amabilidad, el amor y la inteligencia social.

La justicia es una virtud que engloba lo que es moralmente correcto; se basa en la ética, la racionalidad, la ley natural, la religión o los principios de equidad en conjunción con el castigo a las transgresiones de lo que se considera correcto (Konow, 2003). Las fortalezas que engloban la virtud de justicia son la equidad, el liderazgo y el trabajo en equipo.

La virtud de la moderación o templanza está constituida por una serie de fortalezas que protege contra excesos (Peterson, \& Seligman, 2004). Las fortalezas que constituyen la moderación son el perdón, la modestia, la prudencia y la autorregulación.

La virtud de la trascendencia comprende un conjunto de fortalezas que forjan en el individuo conexiones con algo que va más allá 
de él mismo, le provee significado (Pargament, 2009). La trascendencia está conformada por las fortalezas de aprecio por la belleza y la excelencia, la gratitud, la esperanza, el humor y la religiosidad.

\section{Objetivo general del estudio}

Si consideramos las reflexiones y antecedentes arriba vertidos, el presente estudio pretende determinar si las personas que han cometido algún delito presentan rasgos virtuosos $\mathrm{y}$, de ser así, analizar cuáles se manifiestan en la población investigada. Para la determinación de los niveles de virtudes en la muestra se tomaron como referencia los resultados de un estudio previo desarrollado por Corral, Tapia y Ortiz (2015), con participantes de la población general, no reclusos, en la misma ciudad donde se desarrolló la presente investigación. Otro objetivo de la investigación aquí reportada fue probar un modelo estructural de relaciones entre fortalezas y virtudes, que permitiera verificar si la estructura factorial de estos constructos se comporta como en la población general.

\section{Método}

\section{Participantes}

Participaron en el estudio ciento cuatro internos del Centro de Readaptación Social (CERESO), Hermosillo I, en la capital del Estado de Sonora, México. La muestra estuvo constituida por 93 hombres (89.4\%) y 11 mujeres $(10.6 \%)$. La edad promedio de los participantes fue de $35.13(D E=10.5)$ años con una escolaridad promedio de $8.9(D E=2.77)$ años de estudio.

La tabla 1 muestra el tipo de delitos cometidos y el número de participantes que se encontraban recluidos por cada uno de esos tipos de conducta criminal.

Tabla 1

Relación de internos participantes, según delito

\begin{tabular}{lc}
\hline Delito & No. Participantes \\
\hline Robo & 14 \\
Homicidio & 14 \\
Violencia intrafamiliar & 11 \\
Drogas & 14 \\
Secuestro & 13 \\
Violación & 14 \\
Delitos patrimoniales & 10 \\
Lesiones & 14 \\
Total & 104 \\
\hline
\end{tabular}

\section{Instrumentos}

El VIA-S (Peterson, \& Seligman, 2004), en su versión original del idioma español, consta de 240 reactivos; sin embargo, en un estudio previo a esta investigación fue reducido a 120 ítems, conservando las propiedades psicométricas originales, los cuales miden las 24 fortalezas que constituyen las seis virtudes (Ortiz, Corral, Fraijo, \& Tapia, 2015). Decidimos utilizar esta versión reducida. Las opciones de respuesta van de $0=$ "muy diferente a mí", al 4= "muy parecido a mí", con reactivos tales como "Soy 
religioso practicante", "Trato a todo el mundo igual, sea quien sea", "Los derechos de cada uno, sin distinción, son importantes para mí".

\section{Procedimiento}

En un primer momento se solicitó la autorización al secretario de seguridad pública y el permiso al director del CERESO para llevar a cabo la investigación. Posteriormente, por medio del área de Trabajo Social y de Criminología se facilitó el aula y la lista de los internos que podrían participar en el estudio. Se les explicó el objetivo de la investigación y a quienes decidían participar se les entregó el instrumento para llevar a cabo el llenado. Se utilizaron láminas guía para explicar e ilustrar la manera de responder a los internos.

\section{Análisis de datos}

Se calcularon estadísticas univariadas para los reactivos de las sub-escalas empleadas y se obtuvieron indicadores de consistencia interna, a través del coeficiente alfa de Cronbach para cada una de las virtudes. Más tarde, se agruparon las respuestas en índices para cada una de las fortalezas y se promediaron los reactivos que medían cada una de ellas.

Finalmente, se especificó y probó un modelo estructural en el que se formaron factores de primer orden: las fortalezas, los cuales a su vez sirvieron para formar un Factor de Segundo Orden (FSO): virtudes.

\section{Resultados}

La tabla 2 muestra las medias y la consistencia interna de las virtudes y las fortalezas. En todos los casos, los índices de consistencia interna fueron iguales o mayores a .82. Respecto a las seis virtudes, en un rango de respuesta que iba de 0 a 4 , la justicia produjo el valor de media más alto $(M=3.32 ; D E=.59)$, siguiéndole la trascendencia $(M=3.27 ; D E=.52)$, la humanidad $(M=3.25 ; D E=.56)$, el valor $(M=3.19 ; D E=$ .49) y, finalmente, la sabiduría $(M=2.99 ; D E=$ .56) (ver Tabla 2). En el estudio de Corral et al. (2015), con población general, se encontraron medias similares, incluso ligeramente menores a las que produjeron los reclusos de la muestra aquí investigada: (medias de sabiduría $=2.73$, valor $=2.82$, humanidad $=2.93$, trascendencia $=$ 2.83 , justicia $=2.96$, moderación $=2.76$ ).

En el caso de las fortalezas, los índices de medias más altos fueron para esperanza $(M=3.58)$, gratitud $(M=3.54)$, el entusiasmo $(M=3.43)$, trabajo en equipo $(M=3.42)$, amor $(M=3.37)$ y amabilidad $(M=3.37)$. Los valores más bajos fueron para amor por aprender $(M=$ $2.69)$, modestia $(M=2.81)$, perspectiva $(M=$ $2.85)$ y, por último, el aprecio por la belleza y la excelencia $(M=2.94)$. 
Tabla 2

Estadísticas univariadas y consistencia interna de las virtudes y fortalezas

\begin{tabular}{|c|c|c|c|c|c|}
\hline & Mín & Máx & Media & $\mathrm{DE}$ & Alfa \\
\hline \multicolumn{3}{|l|}{ SABIDURÍA } & 2.99 & 0.56 & 0.88 \\
\hline Creatividad & 1.4 & 4 & 3.07 & 0.65 & \\
\hline Curiosidad & 1.4 & 4 & 3.23 & 0.66 & \\
\hline Amor por aprender & 0 & 4 & 2.69 & 0.93 & \\
\hline Mente abierta & 0 & 4 & 3.09 & 0.75 & \\
\hline Perspectiva & 1.6 & 4 & 2.85 & 0.62 & \\
\hline \multicolumn{3}{|l|}{ VALOR/CORAJE } & 3.19 & 0.49 & 0.84 \\
\hline Entusiasmo & 1.4 & 4 & 3.43 & 0.55 & \\
\hline Autenticidad & 1.2 & 4 & 3.06 & 0.67 & \\
\hline Persistencia & 1.6 & 4 & 3.27 & 0.56 & \\
\hline Valentía & 0.8 & 4 & 3.00 & 0.66 & \\
\hline \multicolumn{3}{|l|}{ HUMANIDAD } & 3.25 & 0.56 & 0.82 \\
\hline Amabilidad & 1 & 4 & 3.36 & 0.62 & \\
\hline Amor & 1 & 4 & 3.37 & 0.67 & \\
\hline Inteligencia social & 0.8 & 4 & 3.01 & 0.68 & \\
\hline \multicolumn{3}{|l|}{ TRASCENDENCIA } & 3.27 & 0.52 & 0.87 \\
\hline \multicolumn{2}{|c|}{ Aprecio belleza/excelencia 1} & 4 & 2.94 & 0.75 & \\
\hline Esperanza & 1.6 & 4 & 3.58 & 0.53 & \\
\hline Religiosidad & 1 & 4 & 3.10 & 0.76 & \\
\hline Gratitud & 1.6 & 4 & 3.54 & 0.55 & \\
\hline Humor & 1.2 & 4 & 3.19 & 0.67 & \\
\hline \multicolumn{3}{|l|}{ JUSTICIA } & 3.32 & 0.59 & 0.87 \\
\hline Trabajo en equipo & 1.6 & 4 & 3.42 & 0.62 & \\
\hline Liderazgo & 1.2 & 4 & 3.28 & 0.73 & \\
\hline Equidad & 1.6 & 4 & 3.25 & 0.65 & \\
\hline \multicolumn{3}{|c|}{ MODERACIÓN/TEMPLANZA } & 3.09 & 0.58 & 0.86 \\
\hline Autorregulación & 1.2 & 4 & 3.13 & 0.68 & \\
\hline Modestia & 0.25 & 4 & 2.81 & 0.79 & \\
\hline Perdón & 0.8 & 4 & 3.27 & 0.74 & \\
\hline Prudencia & 1.2 & 4 & 3.14 & 0.70 & \\
\hline
\end{tabular}


La figura 1 presenta los resultados del modelo estructural, muestra que los factores de segundo orden denominados virtudes se conforman a partir de las altas y significativas interrelaciones entre los constructos de primer orden, reconocidos como fortalezas, los cuales cargan alta y significativamente en el factor de segundo orden. En los indicadores de bondad de ajuste se observa que el valor de la $X^{2}$ fue de 411.17 con 248 grados de libertad, asociada a una probabilidad de $\mathrm{p}<.001$. Los indicadores prácticos de bondad de ajuste resultantes fueron: Bentler-Bonett Non-Normed Fit Index (BBNNFI) de .90, Comparative Fit Index (CFI) de .91 y el RMSEA de .08.

Se observa que, aunque la $X^{2}$ resultó ser significativa, el modelo presenta bondad de ajuste práctica según, lo evidencian los indicadores $B N N F I$ y $C F I$, así como el RMSEA. Esto implica que el modelo se ajusta adecuadamente a los datos.

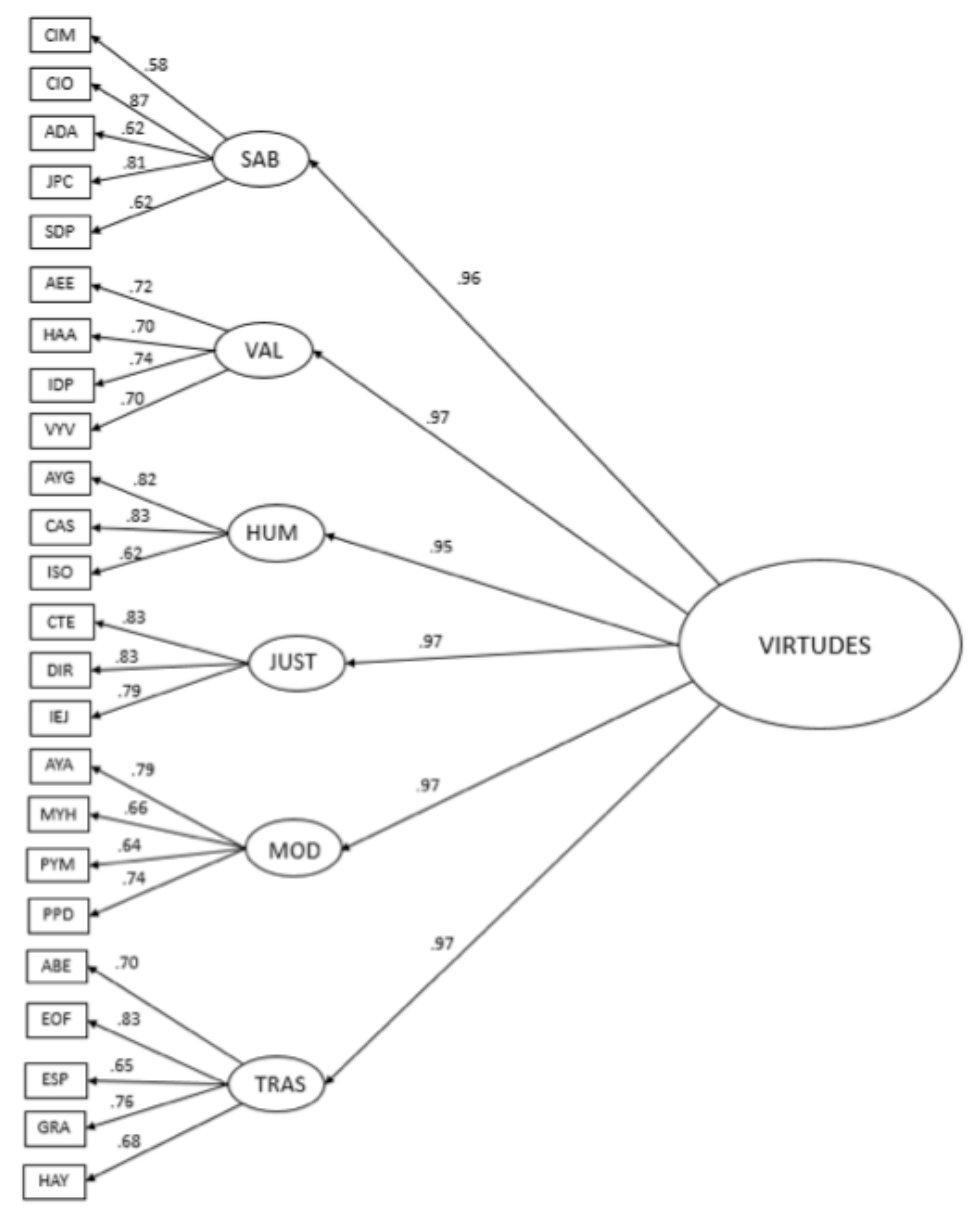

Figura 1. Modelo estructural de las relaciones entre virtudes y fortalezas psicológicas. Índices de bondad de ajuste: $X^{2}=411.17(248 \mathrm{gl}), \mathrm{p}=.000, B B N$ $N F I=.90, C F I=.91, R M S E A=.08$ 


\section{Discusión}

Con los resultados obtenidos en la presente investigación se concluye que los internos en instituciones penitenciarias poseen virtudes y fortalezas de carácter similares a los del resto de la población general. La estructura factorial del modelo de relaciones entre fortalezas y virtudes también comprobó que esas relaciones se comportan como lo hacen en la población general. El modelo mostró relaciones altas y significativas entre las fortalezas y las virtudes particulares, e interrelaciones también altas y significativas entre las virtudes particulares y el constructo de virtudes de orden superior. Estos rasgos son reconocidos universalmente y en la mayoría de las culturas como características positivas (Peterson, \& Seligman, 2004). De acuerdo con nuestros resultados, el que los individuos investigados se encuentren recluidos dentro de un sistema penitenciario no los hace diferentes del resto de las personas en términos de la posesión de dichos rasgos de personalidad. Esos resultados arrojaron que, en la mayoría de las fortalezas de carácter, la media se inclinaba por la opción "parecido a mí".

Aunquea lolargo del estudio delapersonalidad del individuo que cometió un delito, se le ha caracterizado y asociado con factores negativos de personalidad y carencia de virtudes, con los resultados aquí obtenidos resulta pertinente señalar que esos individuos no son, al menos en dichos rasgos, distintos a los demás. Pero entonces, esto nos lleva a preguntarnos: si ellos poseen dichos rasgos positivos de personalidad, ¿por qué no los desarrollan para el "bien” social, de acuerdo con los criterios de la comunidad en general?

Parte de la posible respuesta a esta pregunta es, quizás, que esto se deba a una moderación producida por factores ambientales, de manera que las personas utilizan sus fortalezas de carácter para actuar "positivamente" en un tipo de ambientes, y para comportarse "negativamente" en otros escenarios; otra posibilidad es que las respuestas de los participantes en el estudio se hayan visto influidas por la deseabilidad social. Con respecto a esta segunda opción, dado que el instrumento utilizado es un auto-reporte, a pesar de ser de amplio uso y validado en la población mexicana, éste provocará dudas con respecto a la veracidad de las respuestas. Es probable que la información proporcionada por los participantes tenga un componente de exageración, lo que produciría respuestas similares a las que proporcionan individuos de la población general. Con respecto a la primera opción (el ambiente como moderador de la relación entre virtudes y comportamiento antisocial o prosocial), en años recientes se ha pretendido indagar factores que consideran todos aquellos elementos del entorno no diseñados de manera deliberada, es decir, aquellos elementos que se encuentran en el ambiente y "proveen" o estimulan al individuo y a su vez protegen de o instigan la manifestación de las conductas antisociales e incluso delictivas (Sobral, Romero, Luengo, \& Marzoa, 2000).

El ambiente juega un papel fundamental para la manifestación de ciertos comportamientos, 
ya que en ocasiones las personas se ven involucradas en actos antisociales debido a que son "forzadas" por las demandas que el contexto les impone. Por ejemplo, vivir en un ambiente peligroso e inestable estimula conductas agresivas, de riesgo y lesivas para otros; en cambio, vivir en un entorno pacífico y estable estimula comportamientos prosociales (Brumbach, Figueredo, \& Ellis, 2009). Es decir, quienes se comportan de manera antisocial y presentan comportamientos “desajustados" (para la población general), en muchas ocasiones solamente están cumpliendo con los requerimientos que el medio les exige (delinquir). Lo anterior invita a indagar en aquellas características del medio ambiente que influyen en la manifestación de conductas antisociales y el uso de fortalezas de carácter para desarrollar esas conductas. En este sentido, las fortalezas y virtudes funcionarían como herramientas psicológicas adaptativas que le permiten al individuo ajustarse a las condiciones y exigencias del entorno inmediato. No obstante, a pesar de la lógica de esta explicación, se requiere una prueba empírica de la misma sin llegar al reduccionismo, pues generalmente el comportamiento humano es multifactorial, según la situación y la persona.

En espera de esta verificación, de manera inmediata la conclusión más importante del presente estudio es que las personas recluidas en centros penitenciarios poseen niveles similares de virtudes y fortalezas de carácter a los de aquellas que no se encuentran detenidas por la comisión de actos delictivos.

\section{Referencias}

Beltri, R. T., \& Fuentes, A. C. (2008). Psicopatía: una entidad clínica controvertida pero necesaria en psiquiatría forense. Revista Española de Medicina Legal, 34, 25-35.

Brumbach, B. H., Figueredo, A. J., \& Ellis, B. J. (2009). Effects of harsh and unpredictable environments in adolescence on development of life history strategies". Human Nature, 20, $25-51$.

Corral, V., Frías, M., Gaxiola, J., Tapia, C., Fraijo, B., \& Corral, N. (2014). Ambientes Positivos. Ideando entornos para el bienestar humano y la calidad ambiental. México: Pearson.

Corral, V., Tapia, C., \& Ortiz, A. (2015). On the relationship between character strengths and sustainable behavior. Environment \& Behavior, 47, 877-901.

Corral, V., Tapia, C., Ortiz, A., \& Fraijo, B. (2013). Las virtudes de la humanidad, justicia y moderación y su relación con la conducta sustentable. Revista Latinoamericana de Psicología, 45, 363-374.

Dahlsgaard, K., Peterson, C., \& Seligman, M. (2005). Shared virtue: The convergence of valued human strengths across culture and history. Review of General Psychology, 9, 203-213.

Escrivá, M. V. M., García, P. S., \& Navarro, M. D. F. (2002). Procesos cognitivos y 
emocionales predictores de la conducta prosocial y agresiva: la empatía como factor modulador. Psicothema, 14, 227-232.

Fowers, B. J. (2005). Virtue and psychology: Pursuing excellence in ordinary practices. Washington, DC: APA.

Gacharná, F. I. (2009). Hacker ético vs. delincuente informático: Una mirada en el contexto colombiano. Revista Inventum, 6, 46-49.

Giménez, A., Ojea, H., Nieto, L., Martínez, J., \& Fernández, S. (2012). Estructuras y liderazgo en cuatro redes españolas dedicadas al tráfico de drogas. Revista Española de Investigación Criminológica, 10, 1-32.

Greeff, E. (1950). Criminogénesis. Trabajo presentado en el Congreso Internacional de Criminología. París.

Hesnard, A. (1963). Psicología del crimen. Barcelona: Editorial Zeus.

Hurwttz, S. (1956). Criminología. Barcelona: Ediciones Ariel.

Kohlbert, L. (1969). Stages in the development of moral thought and Action. New York: Holt, Rinehart and Winston.

Konow, J. (2003). Which is the fairest one of all? A positive analysis of Justice Theories. Journal of Economic Literature, 61, 11881239.

Landazabal, M. (2005). Conducta antisocial durante la adolescencia: Correlatos socioemocionales, predictores y diferencias de género. Psicología Conductual, 13, 197-215.
Linley, P. (2009). Positive Psychology (History). En S. Lopez (Ed.) The Encyclopaedia of Positive Psychology (pp.742-146). Chichester, UK: Wiley-Blackwell.

Maqueda, M. (2010). Aproximación a la violencia juvenil colectiva desde una criminología crítica (bandas, tribus y otros grupos de calle). Revista de Derecho Penal y Criminología, 4, 271-331.

Marchiori, H. (2004). Criminología. La víctima del delito. México: Editorial Porrúa.

Moffitt, T. (1997). Adolescence-limited and life course persisting offending: A complementary par of developmental theories. En T. Thornberry (Ed.) Advances in Criminology Theory. Developmental Theories and Crime and Delinquency (pp. 11-54). Londres: Transaction Press.

National Research Council and Institute of Medicine. (2001). Juvenile Crime, Juvenile Justice. Washington, DC: National Academy Press.

Ortiz, A., Corral, V., Fraijo, B., \& Tapia, C. (2015, octubre). Validación de una versión reducida del inventario de virtudes $y$ fortalezas. Trabajo presentado en el XXIII Congreso Mexicano de Psicología. Cancún, México.

Pargament, K. (2009). Spirituality. En: S. López (Ed.), The Encyclopedia of Positive Psychology (pp. 928- 932). Chichester, UK: Wiley-Blackwell. 
Peterson, C., \& Seligman, M. (2004). Character strengths and virtues: a handbook and classification. Washington, DC: American Psychological Association.

Pizarro, D., \& Salovay, P. (2002). On being and becoming a good person: The role of emotional intelligence in moral development and behavior. En J. Aronson (Ed.) Improving Academic Achievement: Impact of Psychological Factors on Education (pp. 247-266). San Diego, CA: Academic Press.

Pinatel, J. (1979). La Sociedad Criminógena. Madrid: Editorial Aguilar.

Resten, R. (1963). Caracterología del Criminal. Barcelona: Ed. Miracle.

Romero, E. (2001). El constructo psicopatía en la infancia y la adolescencia: del trastorno de conducta a la personalidad antisocial. Anuario de psicología/The UB Journal of psychology, 32, 25-49.

Romi, J. C. (1995). Reflexiones sobre la conducta sexual delictiva. Revista Argentina de Psiquiatría Forense, Sexología y Praxis, 2, 117-130.

Seligman, M. (2011). Flourish $-A$ new understanding of happiness and wellbeingand how to achieve them. Londres: Nicholas Brealey Publishing.

Seligman, M., \& Csikszentmihalyi, M. (2000). Positive psychology: An introduction. American Psychologist, 55, 5-14.

Sharma, N., Prakash, O., Sengar, K., Shaudhury, S., \& Sing, A. (2015). The relation between emotional intelligence and criminal behavior: A study among convicted criminals. Industrial Psychiatry Journal, 24, 54-58.

Sobral, J., Romero, E., Luengo, A., \& Marzoa, J. (2000). Personalidad y conducta antisocial: amplificadores individuales de los efectos contextuales. Psicothema, 12, 661-670.

Temperini, M. G. (2014). Delitos informáticos en Latinoamérica: un estudio de derecho comparado. En XLIII Jornadas Argentinas de Informática e Investigación Operativa (43JAIIO)-XIV Simposio Argentino de Informática y Derecho (SID). Buenos Aires.

Vera, B. (2006). Psicología Positiva: Una nueva forma de entender la Psicología. Papeles del Psicólogo, 27, 3-8.

Recibido el 14 de julio de 2016

Revisado el 10 de octubre de 2016

Aceptado el 10 de octubre de 2016 